\title{
Examining the Relationship Between Perceived Autonomy Support and Age in the Context of Rehabilitation Adherence in Sport
}

\author{
Andrew R. Levy \\ University of Leeds
}

\author{
Remco C. J. Polman \\ University of Hull
}

\author{
Erika Borkoles \\ Leeds Metropolitan University
}

\begin{abstract}
Objective: To investigate the relationship between perceived autonomy support, age, and rehabilitation adherence among sports-related injuries. Design: A prospective design measuring adherence over the entire rehabilitation period (8-10 weeks) was employed. Participants: 44 men and 26 women aged between 18 and 55 years took part in this study $(M=32.5$ years, $S D=10.2)$. Method: Adherence was monitored via an observational measure of clinic-based adherence, a self-report measure of home-based adherence, and monitoring of attendance at rehabilitation sessions. Perceived autonomy support of participants was assessed during the last week of their rehabilitation. Results: High autonomy support provided by the physical therapist was related to better clinic-based adherence and attendance but not to home-based adherence. Age was related to all adherence indices and moderated the relationship between perceived autonomy support and clinic-based rehabilitation adherence. Conclusion: Findings indicate the importance of physical therapists providing an autonomous-supportive rehabilitation environment, particularly among older individuals.
\end{abstract}

Keywords: age, rehabilitation adherence, autonomy

Few issues can unify medical and health care professions to the extent that nonadherence can (Meichenbaum \& Turk, 1987). This commonality has given rise to a substantial amount of literature that has examined adherence issues across both medical and health domains. More recently, researchers have turned their attention toward adherence issues that concern sport-injury rehabilitation programs. This interest may have emanated from numerous physical therapists, athletic trainers, and sports medicine physicians identifying nonadherence as a frequently reoccurring problem with athletes (Brewer, 1999). Estimates suggest adherence rates for clinic-based rehabilitation among an athletic population to be as low as 40\% (Daly, Brewer, Van Raalte, Petitpas, \& Sklar, 1995). Similarly, Taylor and May (1996) reported less than adequate adherence rates, ranging between $54 \%-60 \%$, for athletes undertaking home-based rehabilitation. It must be noted that a standardized definition of sport-injury rehabilitation adherence is difficult to achieve due to the extensive variability in activities across sport injuries and rehabilitation programs (Brewer, 1999). However, there is a general consensus that adherence in the context of sport rehabilitation concerns a common set of broad behaviors that involve (a) adhering to instructions to engage in appropriate restriction of physical activity, (b) completing home rehabilitation

Andrew R. Levy, Centre for Sport and Exercise Science, University of Leeds; Remco C. J. Polman, Department of Sport, Health and Exercise Science, University of Hull; Erika Borkoles, Carnegie Faculty of Sport \& Education, Leeds Metropolitan University.

Correspondence concerning this article should be addressed to Andrew R. Levy, PhD, Centre for Sport and Exercise Science, University of Leeds, Worsley Building, Leeds LS2 9JT, UK. E-mail: A.Levy@Leeds.ac.uk exercises, (c) complying with treatment (i.e., ice/heat therapy), and (d) attending and actively participating in clinic-based rehabilitation exercises.

Previous sport-injury rehabilitation adherence studies have identified various situational factors associated with augmented adherence to sport-injury rehabilitation regimens. For example, several retrospective studies have found that adherent athletes experienced greater social support (Byerly, Worrell, Gahimer, \& Domholdt, 1994; Fisher, Domm, \& Wuest, 1988), convenient rehabilitation scheduling (Fields, Murphey, Horodyski, \& Stopka, 1995; Fisher et al., 1988), and perceived themselves to work harder (Fisher et al., 1988) than their nonadherent counterparts. More recent prospective research has also found social support (Brewer et al., 2000), alongside beliefs regarding the efficacy of treatment (Brewer et al., 2003b), to be predictive of clinic-based rehabilitation adherence.

In addition to perceived control (Folkman, 1984) and social support (Reis \& Franks, 1994), there is a key set of psychological processes, namely the process of autonomy, that has largely been ignored by the adherence literature. Deci and Ryan (1985, 1995) referred to autonomy as the need to express one's authentic self, that is, the source of one's actions. They postulated that being autonomous and having autonomy-supportive relationships underlies the self-determination process. The self-determination theory of human motivation (Deci \& Ryan, 1985) proposes that three primary psychological needs exist: autonomy, relatedness, and competence. Self-determined behavior is said to be based on a process of internalization, which concerns the degree to which an individual's basic psychological needs are satisfied (Deci \& Ryan, 1985). Opportunities to experience oneself as autonomous are said 
to be facilitated by contexts that are autonomy supportive (Deci \& Ryan, 1987, 1991). Of main interest to the present study, therefore, is the notion of autonomy support in the rehabilitation process and the extent to which medical personnel enable patients to experience a degree of autonomy. Accordingly, practitioners need to respect the patients in social and medical interactions, allowing their patients freedom of expression and enabling them to be true to their inner values and desires (Skinner \& Edge, 2002). To date, the practitioner's interpersonal style of being autonomy supportive or controlling has not been examined extensively within a rehabilitation-adherence capacity, yet it is viewed to be a very important determinant of health behavior because it can be taught to practitioners (Williams \& Deci, 1996). The key variables of being autonomy supportive in a rehabilitation context concern acknowledging patient feelings and also allowing the patient to express views and opinions of their injury while the practitioner gives significance to these in the decision-making and problem-solving process during treatment (Williams, Gagné, Ryan, \& Deci, 2002).

From an emotional perspective, a construct linked to autonomy support that has received attention within the medicine literature concerns empathy. Physicians' empathetic behaviors encapsulate an accurate understanding and acceptance of a patient's feelings or concerns (Frankel, 1995). According to Newton et al. (2000), patient perceptions of whether their physician is demonstrating empathy can impact treatment behavior and subsequent outcomes. A study by Kim, Kaplowitz, and Johnston (2004) found that Korean patients' $(n=550)$ perceptions of physicians' empathetic behavior were directly related to treatment compliance and satisfaction. This finding concurs with a number of earlier reviews (Becker \& Maiman, 1975; Garrity, 1981; Haynes, 1976) that have concluded there to be a significant relationship between physician empathy and adherence to treatment regimens. Further to this, Zachariae et al. (2003) found that oncology outpatients $(n=454)$ who perceived greater physician empathy had greater confidence in their ability to cope with disease-related treatments and symptoms. Given the importance of patients' judgments regarding their physician's empathy, Silvester, Patterson, Koczwara, and Ferguson (2007) concluded that empathetic behaviors should be an important feature of training for physicians and other professional disciplines where empathy is deemed to be an integral part of treatment success.

Numerous authors within the sport-injury rehabilitation literature have alluded to but not investigated the importance for physical therapists to provide an autonomy-supportive environment in order to promote favorable rehabilitation behavior. For example, Taylor and Marlow (2001) recommended that the physical therapist should empower the athlete by encouraging ownership of their rehabilitation program, listening to athletes' needs, and including them whenever possible in the decision making about their treatment plan. As such, it is intuitive to regard autonomy support provided by the physical therapist to be associated with greater adherence to sport-injury rehabilitation programs. However, as far as we are aware, no studies to date have considered the implications of an autonomy-supportive environment provided by the physical therapist upon rehabilitation-adherence behavior within the domain of sport injuries. Accordingly, the first purpose of this investigation was to examine the relationship between adherence to sport-injury rehabilitation programs and athlete perceptions of autonomy supportiveness provided by their physical therapist. It was hypothesized that individuals who hold higher perceptions of autonomy support would display greater adherence across all measured indices of rehabilitation adherence.

According to Levy, Polman, Clough, and McNaughton (2006), a notable limitation regarding the majority of sport-injury rehabilitation adherence studies to date is their neglect of moderating variables that may influence the direction and/or strength of the relationships between psychological variables (independent factors) and rehabilitation adherence (observed phenomenon). Weiss (2003) acknowledged that a key moderating variable requiring attention in the context of sport-injury rehabilitation is age. Her rationale for this was based on evidence from developmental sport-psychology research that has found both younger and older adults to differ in their self-perceptions, social influences, emotional responses, motivations, and self-regulation with regard to sport and exercise participation. Weiss (2003) concluded that these differences may be extrapolated to rehabilitation contexts and thus warranted empirical attention. In response to this, Brewer et al. (2003a) examined whether the relationship between selected psychological variables and rehabilitation adherence among postoperative anterior cruciate ligament athletes differed as a function of age. Findings revealed that both self-motivation and social support were positively associated with home-based adherence for older adults, whereas athletic identity was found to be predictive of home-based adherence with younger participants. Brewer et al. (2003a) concluded that age may be a salient factor when organizing sport-injury rehabilitation programs and recommended future research to consider this variable. Consequently, the second purpose of this investigation was to examine the direct impact of age upon rehabilitation adherence and investigate whether the relationship between perceived autonomy support and rehabilitation adherence differs as a function of age. Specifically, it was hypothesized that age would be significantly related to all three indices of rehabilitation adherence and would also moderate the relationship between perceived autonomy support and measures of rehabilitation adherence.

\section{Method}

\section{Participants}

The present study consisted of 70 (44 men and 26 women) participants aged between 18 and 55 years $(M=32.5$ years, $S D=$ 10.2) and were recruited from four private physiotherapy clinics. Participants indicated that they were either competitive athletes $(31 \%)$ or recreational athletes $(69 \%)$ and had sustained a tendonitis-related overuse injury in which no prior surgery had been necessary. Notably, tendonitis injuries sustained were mainly located at the ankle $(41 \%)$, knee $(28 \%)$, shoulder $(20 \%)$, or elbow $(11 \%)$. The duration of participants' rehabilitation ranged from 8 to 10 weeks. In accordance with ethical guidelines, ethical approval was obtained from the University of Hull Ethics Committee. In addition, informed consent was provided by all participants.

\section{Procedure}

At their first physiotherapy appointment, participants were required to complete a questionnaire assessing demographic and injury-related information. At each clinic rehabilitation session 
(two sessions per week), the physical therapist recorded participants' attendance and completed the Sport Injury Rehabilitation Adherence Scale (SIRAS; Brewer et al., 2002). Alongside this, participants were instructed to monitor their adherence to homebased rehabilitation (one session per week) via a self-report measure. Upon their last clinic rehabilitation session, participants completed the Health Care Climate Questionnaire (HCCQ; Williams, Grow, Freedman, Ryan, \& Deci, 1996), which assessed perceived autonomy support.

\section{Measures}

Perceived autonomy support. This construct was assessed with the 15-item HCCQ, which was initially designed for patients to rate autonomy supportiveness of their physician. In the present study, the word "physician" was replaced with "physiotherapist" in all items. This is consistent with previous studies that had differing providers of autonomy support (Williams, Rodin, Ryan, Grolnick, \& Deci, 1998). Ratings for the HCCQ were made on 7-point Likert-type scales with endpoints ranging from 1 (strongly disagree) to 7 (strongly agree). A total perceived autonomy support score was derived by summing each of the HCCQ items. Exploratory factor analysis conducted on the 15-item HCCQ has revealed this measure to have a one-factor solution on which all factor loadings were above .74 (Williams, Grow, et al., 1996). Further to this, Cronbach's coefficient alphas have been demonstrated to be consistently above .80 (Williams, Grow, et al., 1996; Williams et al., 1998). This suggests that the 15-item HCCQ has sound psychometric properties.

Adherence. A multifaceted approach toward the assessment of adherence was employed. First, attendance at rehabilitation appointments was calculated by dividing the number of rehabilitation sessions attended by the number of prescribed rehabilitation visits. Secondly, the SIRAS was utilized to assess adherence during clinic-based rehabilitation sessions. This instrument requires the physical therapist to evaluate patients' behavior on a 5-point Likert scale with regard to (a) intensity with which participants completed their prescribed exercises (end points: $1=$ minimum effort, $5=$ maximum effort), (b) the frequency with which participants followed instructions (end points: $1=$ never, $5=$ always), and (c) their receptiveness to changes toward the program (end points: $1=$ very unreceptive, $5=$ very receptive). A total clinic-based rehabilitation-adherence score was derived by summing each of the SIRAS responses for each item. The SIRAS has good internal consistency, ranging from $\alpha=.81$ to $\alpha=.86$ (Shaw, Williams, \& Chipchase, 2005), and has also been reported to have good test- retest reliability over a 1-week period (intraclass correlation coefficient $=0.77$; Brewer et al., 2000). In addition, Brewer et al. (2002) demonstrated the construct validity of the SIRAS with a heterogeneous sample of 43 participants. They found a significant linear relationship $(p<.001)$ between SIRAS scores and observed video footage that depicted minimal, moderate, and maximal clinic-based rehabilitation adherence. A self-report measure of homebased rehabilitation adherence, as recommended by Bassett (2003), was utilized. This required participants to indicate on a 5-point Likert scale ranging from 1 (not at all) to 5 (as advised) the extent to which they had (a) completed recommended home exercises, (b) refrained from undertaking activity that could harm injury, and (c) applied home cryotherapy (icing). A total homebased rehabilitation-adherence score was derived by summing each of the self-report responses for each item.

\section{Statistical Analyses}

Cronbach's alphas and descriptive statistics were calculated on all study variables. To determine the effects of perceived autonomy support and age upon the three measures of rehabilitation adherence, we employed a two-way between-subjects multivariate analysis of variance. The between-subject factors, perceived autonomy support (high vs. low) and age (young vs. old), both had two levels. In order to establish where significant differences existed, we conducted a follow-up univariate analysis of variance using a Bonferroni adjusted alpha level of .017. Given that the independent variable (perceived autonomy support) and the moderating variable (age) are both categorical in nature, Baron and Kenny (1986) stipulated that the interaction effect produced via an analysis of variance is a suitable indicator of moderation. Univariate and multivariate normality were assessed using skewness/ kurtosis values and Mahalanobis distances, respectively.

\section{Results}

\section{Descriptive Statistics}

Means, standard deviations, and Cronbach's alpha reliability coefficients $(\alpha)$ for the HCCQ and rehabilitation adherence are displayed in Table 1. Notably, measures exhibited good internal consistency, with all alphas exceeding .80. None of the variables violated univariate or multivariate normality.

Table 1

Bivariate Correlations, Descriptive Statistics, and Skewness/Kurtosis Values for Perceived Autonomy Support and RehabilitationAdherence Indices

\begin{tabular}{llllrrrrr}
\hline \multicolumn{1}{c}{ Variable } & 1 & 2 & 3 & 4 & $M$ & $S D$ & Skewness & Kurtosis \\
\hline PAS & $(.87)$ & & & & 70.24 & 14.15 & -.09 & -0.68 \\
Clinic adherence & $.34^{*}$ & $(.93)$ & & & 262.83 & 96.91 & -.65 & -1.21 \\
Home adherence & .03 & $.87^{*}$ & $(.93)$ & & 90.03 & 27.05 & -.18 & -1.43 \\
Attendance & $.38^{*}$ & $.79^{*}$ & $.55^{*}$ & - & 90.56 & 8.57 & -.82 & -0.15 \\
\hline
\end{tabular}

Note. Cronbach's alphas are presented on the diagonals. The dash indicates that the alpha for that construct was unobtainable. PAS $=$ perceived autonomy support.

${ }^{*} p<.01$. 


\section{Relationship Between Perceived Autonomy Support and Rehabilitation Adherence}

Multivariate analysis revealed a significant main effect for perceived autonomy-support groups and the three measures of rehabilitation adherence, $F(3,64)=15.59, p<.001$, Wilks's $\lambda=.58$, partial $\eta^{2}=.42$. When adherence measures were considered separately, via univariate analysis, only clinic-based adherence, $F(1,66)=23.73, p<.01$, partial $\eta^{2}=.26$, and attendance, $F(1$, $66)=23.96, p<.01$, partial $\eta^{2}=.27$, reached significance. An inspection of the mean scores $( \pm S D)$ indicated that those who perceived high autonomy support adhered better to clinic-based rehabilitation (305.44 \pm 39.18) and attended more frequently $(94.88 \pm 4.63)$ than did those who reported low autonomy support (clinic-based rehabilitation: $226.95 \pm 115.48$; attendance: $86.92 \pm$ 9.46).

\section{Relationship Between Age and Rehabilitation Adherence}

There was a significant multivariate effect for age, $F(3,64)=$ $3.58, p=.019$, Wilks's $\lambda=.86$, partial $\eta^{2}=.14$, with significant differences being observed in all three dependant variables. Specifically, univariate analysis indicated there were significant agegroup differences for clinic-based rehabilitation, $F(1,66)=8.89$, $p=.004$, partial $\eta^{2}=.12$, home-based rehabilitation, $F(1,66)=$ $8.50, p=.005$, partial $\eta^{2}=.11$, and attendance, $F(1,66)=7.88$, $p=.007$, partial $\eta^{2}=.11$. Mean scores indicated that compared to older individuals (attendance: $87.76 \pm 9.76$; clinic-based rehabilitation: $228.12 \pm 100.95$; home-based rehabilitation: $78.56 \pm$ 26.48), younger individuals attended their rehabilitation sessions more frequently $(92.11 \pm 7.51)$ and had better clinic-based $(282.11 \pm 90.02)$ and home-based $(96.40 \pm 25.46)$ adherence.
Relationship Between Perceived Autonomy Support, Age, and Rehabilitation Adherence

Multivariate analysis for both perceived autonomy support and age indicated a significant interaction effect, $F(3,64)=3.67, p=$ .017 , Wilks's $\lambda=.85$, partial $\eta^{2}=.15$. Follow-up univariate analysis established that only clinic-based rehabilitation adherence reached a significant interaction, $F(1,66)=7.03, p=.010$, partial $\eta^{2}=.01$. Further inspection of mean scores, displayed in Figure 1 , revealed that the younger group who perceived high autonomy support had the greatest adherence to clinic-based activities (308.16 \pm 18.59$)$, whereas their older counterparts who had poorer perceptions of autonomy support $(148.67 \pm 23.39)$ were found to have the lowest clinic-based rehabilitation adherence.

\section{Discussion}

The main aims of the present study were to investigate the relationship between perceived autonomy support and adherence to sport-injury rehabilitation programs and to establish whether the latter relationship was moderated by age. In addition to this, the direct impact of age upon rehabilitation adherence was also examined. Findings revealed that high perceptions of autonomy supportiveness provided by the physical therapist led to better clinicbased adherence and attendance at rehabilitation sessions, both of which had moderate effect sizes. Home-based rehabilitation, however, was not associated with perceived autonomy supportiveness. Despite modest effect sizes, age was found to have a direct relationship with attendance and also with both clinic-based and home-based rehabilitation adherence. In particular, younger individuals performed better on all the aforementioned adherence

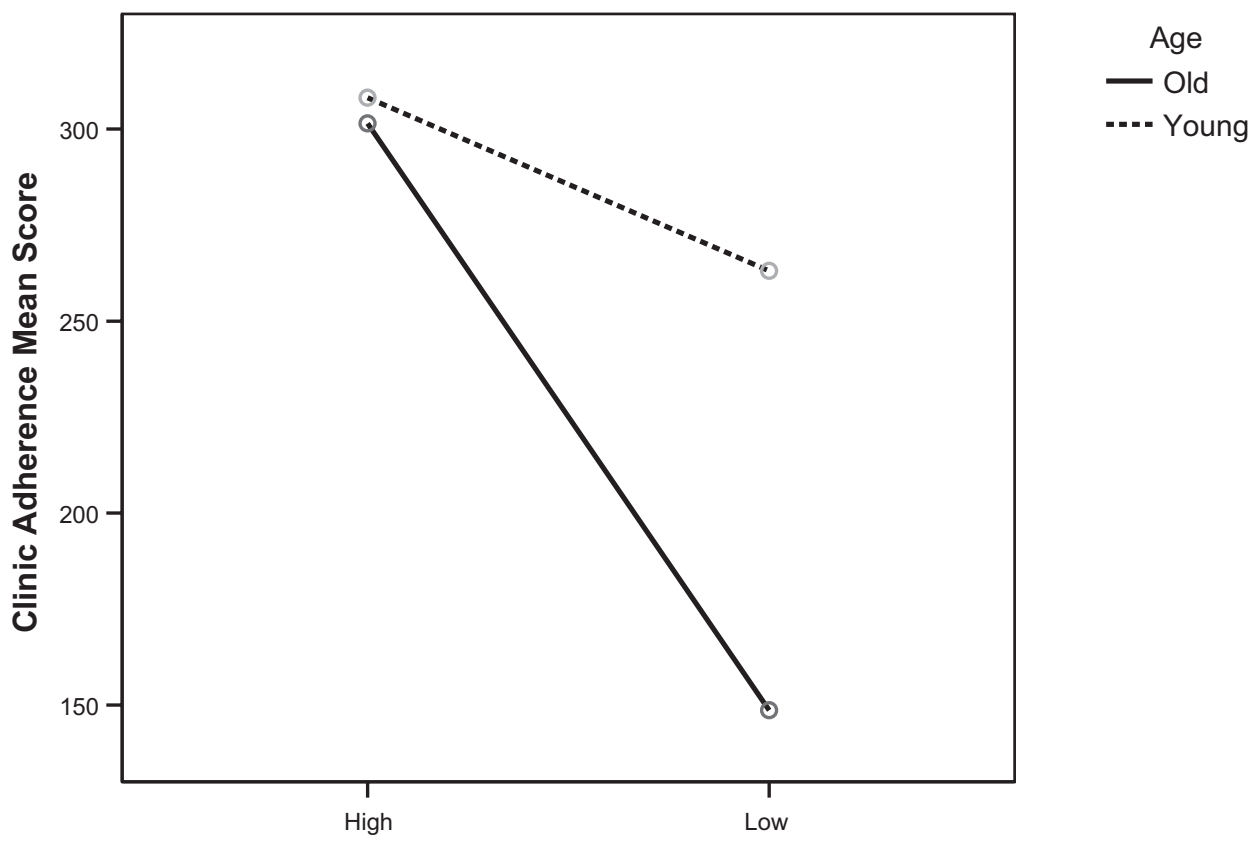

Figure 1. Effect of interaction between perceived autonomy support and age on clinic adherence. 
measures than did their older counterparts. Age also appeared to moderate the perceived autonomy support and clinic-based rehabilitation adherence relationship, albeit that the effect size for this interaction was small. In spite of this, clinic-based adherence was greatest among younger individuals who had higher perceptions of autonomy supportiveness. In contrast, clinic-based adherence was lowest among older individuals who had lower perceptions of autonomy supportiveness. Notably, age did not moderate perceived autonomy support in relation to home-based adherence and attendance.

Complementing the findings of the present study, previous research, as highlighted in the introduction, has found perceived autonomy support to be related with numerous health-related adherent behaviors. One explanation for this, put forward by the self-determination theory, is that autonomy-supportive contexts contribute to the development of autonomous regulations, which are necessary for self-determined behavior. That is, being autonomy supportive can influence patients' intrinsic motivation and identification, which is most beneficial for optimal and continued behavioral engagement. Before this can be confirmed, however, future research is needed to test the mediating effect of perceived autonomy support upon autonomous regulations and rehabilitation adherence in sport.

An implication that can be drawn from the present study is the importance of autonomy support by rehabilitation staff on attendance and clinic-based rehabilitation adherence. This may be achieved by making the effort to explain treatments in a language familiar to the patients, adhering to evidence-based standards, setting limits for patients, making medical treatment recommendations, and by giving feedback. However, these should be conveyed in a nonjudgmental, understanding, and encouraging style (Sheldon, Williams, \& Joiner, 2003), where the patients are listened to, their feelings and emotions are acknowledged, and their personal rehabilitation goals are established with some guidance from the practitioner. A recent longitudinal randomized control trial study on smoking cessation by Williams et al. (2006) showed that perceived autonomy support by practitioners led to increased autonomous and competence motivation in patients, which in turn led to greater abstinence from smoking. They showed the causal role of autonomy support in the internalization of autonomous motivation and perceived competence. An earlier study by Williams et al. (1998) also confirmed that patients' autonomous motivation for adherence does mediate the relationship between patients' perceptions of their doctor's autonomy support and their own medication adherence.

However, there is little empirical basis for improving adherence (Haynes, McKibbon, \& Kanani, 1996), especially concerning physical-therapy treatments. A recent study by Niven (2007) recognized the importance for physical therapists in tailoring rehabilitation to accommodate the needs of athletes in conjunction with involving them in the decision-making process in order to promote rehabilitation adherence, but the study lacked essential theoretical underpinning. Patients are autonomously motivated when they experience choice and volition while behaving (Deci \& Ryan, 1985; Williams et al., 2006). When adhering to physiotherapy treatments, patients are autonomous if they freely choose to do their treatment and prescribed exercises to aid their recovery and improve their future health. Patients may perceive themselves competent when they feel that they have progressed through the treatment and were given specific feedback to help them to achieve their treatment goals. It appears that autonomy support within the health care environment predicts greater autonomous motivation by the patient for specific health-related behaviors, despite controlling for the patient autonomy orientation at a personal level (Williams et al., 2002). Future studies, therefore, should consider the relationship of both autonomy and relatedness upon perceived autonomy support provided by the physical therapist in the context of rehabilitation adherence.

Age is a developmental factor that has been given scant attention within the sport-injury rehabilitation literature (Weiss, 2003). The present study addressed this issue with respect to rehabilitation behavior and found that younger individuals adhered better to rehabilitation than did their older counterparts. One explanation for this put forward by Bandura (2001) suggests that advancing age can be associated with reductions in control beliefs regarding mobility and functional performance. Thus, older individuals might be less efficacious regarding their ability to attend and engage in clinic rehabilitation exercises. Using exercise participants, McAuley, Shaffer, and Rudolph (1995) found that older participants reported lower self-efficacy than younger individuals did. This would suggest that efficacy cognitions may differ as a function of age. Despite previous research identifying self-efficacy to be a key predictor of sport-injury rehabilitation adherence (Brewer et al., 2003b), no research to date has examined the moderating effect of age upon this relationship, thus warranting further attention. In addition to this, future researchers may wish to consider age-related differences in relation to other key variables found to impact sport-injury rehabilitation adherence and functional outcomes. For instance, it is possible that as a result of degenerative processes, older individuals may experience greater pain symptoms, which may negatively impact rehabilitation and subsequent recovery outcomes. A recent study by Brewer et al. (2007) found that among those who had undergone anterior cruciate ligament reconstruction, older participants reported experiencing greater pain than did their younger counterparts. Furthermore, Fisher et al. (1988) found adherent sport participants had higher pain tolerance than did nonadherent individuals. Although this study did not test the moderating effect of age, findings by Woodrow, Friedman, Siegelaub, and Collen (1972) showed that pain tolerance actually decreased with age among a large medical population. As such, it is possible that older individuals may have difficulty adhering to their prescribed rehabilitation modalities due to them having a lower pain threshold. However, before this contention can be confirmed, it is important that future researchers explore the moderating impact of age upon key predictors, such as pain, upon sport-injury rehabilitation adherence.

Another reason for age-related differences with respect to rehabilitation adherence in the present study may be how people feel during and after rehabilitation. Within an exercise capacity, McAuley et al. (1995) found that older participants reported more negative affect than younger individuals did during and after a bout of exercise. Further, affective states have been proposed to influence exercise participation (Ekkekakis, Hall, \& Petruzzello, 2005). In the context of rehabilitation in sport, Daly et al. (1995) found that patients who displayed signs of mood disturbance were more likely to miss scheduled rehabilitation appointments, which could have negative consequences for recovery. Despite Daly et al.'s (1995) finding, their study did not consider age. Therefore, 
future research is required to examine the moderating role of age upon affect and rehabilitation adherence.

The present findings expand Brewer et al.'s (2003a) study exploring age-related differences among psychological factors and rehabilitation adherence. In particular, our study found greater clinic rehabilitation adherence to be associated with younger individuals who reported favorable perceptions of autonomy supportiveness, but the opposite was apparent for older participants. This is an important finding because no studies, to our knowledge, have attempted to test the moderating effect of age upon perceived autonomy support and behavioral involvement. As such, further research is required in order to examine the veracity of our findings. Despite this, a preliminary implication from the present study suggests that individuals of different ages may have differing perceptions of the clinic rehabilitation environment. In particular, rehabilitation staff may need to be aware that older individuals may adhere better to clinic rehabilitation modalities if they receive greater autonomy support. Before this can be confirmed, however, experimental research that manipulates autonomy support and evaluates its effects upon clinic rehabilitation adherence among different injured age groups is warranted.

Although the findings of this study expand knowledge regarding the role of perceived autonomy support and age upon rehabilitation adherence, there are several limitations that should be acknowledged. First, due to the study design, causality cannot be inferred, therefore future research adopting experimental and longitudinal/ repeated-measure designs is required in order to provide stronger support for the findings outlined in the present study. Second, the sample size was relatively small, mainly due to the difficulty of obtaining a large homogeneous sample with regard to injury type, as recommended by Brewer (1999), and the age range among the participants can be considered restrictive. Third, despite the prospective measurement of rehabilitation adherence, perceived autonomy support was measured retrospectively. Brewer (1999) suggested that retrospective measurement limits conclusions that can be drawn from the findings. However, it is possible that a person's perception of autonomy support may vary throughout their course of rehabilitation. Therefore, administering the HCCQ at the end of rehabilitation would have allowed participants to reflect on autonomy support, which may have increased response accuracy. In addition, the HCCQ was completed during the last week of rehabilitation so its retrospective time lag in relation to adherence measures was minimal. Fourth, despite the virtues of using a homogeneous sample in terms of injury type (Brewer, 1999), additional inquiry with different types of injuries and rehabilitation protocols are necessary to determine the extent to which the present findings are representative. Finally, the home-based rehabilitation measure used in this study has not been psychometrically evaluated. However, according to Shaw et al. (2005), research relating to rehabilitation adherence in sport is a relatively new area, and thus few adherence measures have been extensively tested for reliability and validity.

In summary, the findings of the present investigation suggest that both perceived autonomy support and age can impact rehabilitation adherence. In addition, age was found to moderate the relationship between perceived autonomy support and clinic-based rehabilitation adherence. On the basis of these findings, rehabilitation staff needs to be aware of their importance in creating an autonomous-supportive rehabilitation environment, particularly among older individuals. It is important that future research further examines our findings among different athletic populations and injury types.

\section{References}

Bandura, A. (2001). Social cognitive theory: An agentic perspective. Annual Review of Psychology, 52, 1-26.

Baron, R. M., \& Kenny, D. A. (1986). The moderator-mediator variable distinction in social psychological research: Conceptual, strategic, and statistical considerations. Journal of Personality and Social Psychology, 51, 1173-1182.

Bassett, S. F. (2003). The assessment of patient adherence to physiotherapy rehabilitation. New Zealand Journal of Physiotherapy, 31, 60-66.

Becker, M. H., \& Maiman, L. A. (1975). Sociobehavioral determinants of compliance with health and medical care recommendations. Medical Care, 12, 10-24.

Brewer, B. W. (1999). Adherence to sport injury rehabilitation regimes. In S. Bull (Ed.), Adherence issues in sport and exercise (pp. 145-168). Chichester, UK: Wiley.

Brewer, B. W., Avondoglio, J. B., Cornelius, A. E., Van Raalte, J. L., Brickner, J. C., Petitpas, A. J., et al. (2002). Construct validity and interrater agreement of the sport injury rehabilitation adherence scale. Journal of Sport Rehabilitation, 11, 170-178.

Brewer, B. W., Cornelius, A. E., Sklar, J. H., Van Raalte, J. L., Tennen, H., Armeli, S., et al. (2007). Pain and negative mood during rehabilitation after anterior cruciate ligament reconstruction: A daily process analysis. Scandinavian Journal of Medicine and Science in Sports, 17, 520-529.

Brewer, B. W., Cornelius, A. E., Van Raalte, J. L., Petitpas, A. J., Sklar, J. H., Pohlman, M. H., et al. (2003a). Age-related differences in predictors of adherence to rehabilitation after anterior cruciate ligament reconstruction. Journal of Athletic Training, 38, 158-162.

Brewer, B. W., Cornelius, A. E., Van Raalte, J. L., Petitpas, A. J., Sklar, J. H., Pohlman, M. H., et al. (2003b). Protection motivation theory and adherence to sport injury rehabilitation revisited. The Sport Psychologist, 17, 95-103.

Brewer, B. W., Van Raalte, J. L., Cornelius, A. E., Petitpas, A. J., Sklar, J. H., Pohlman, M. H., et al. (2000). Psychological factors, rehabilitation adherence, and rehabilitation outcome after anterior cruciate ligament reconstruction. Rehabilitation Psychology, 45, 20-37.

Byerly, P. N., Worrell, T., Gahimer, J., \& Domholdt, E. (1994). Rehabilitation compliance in an athletic training environment. Journal of Athletic Training, 29, 352-355.

Daly, J. M., Brewer, B. W., Van Raalte, J. L., Petitpas, A. J., \& Sklar, J. H. (1995). Cognitive appraisal, emotional adjustment, and adherence to rehabilitation following knee surgery. Journal of Sport Rehabilitation, 4, 23-30.

Deci, E. L., \& Ryan, R. M. (1985). Intrinsic motivation and self-determination in human behavior. New York: Plenum Press.

Deci, E. L., \& Ryan, R. M. (1987). The support of autonomy and the control of behavior. Journal of Personality and Social Psychology, 53, $1024-1037$.

Deci, E. L., \& Ryan, R. M. (1991). A motivational approach to self: Integration in personality. In R. Dienstbier (Ed.), Nebraska symposium on motivation: Vol. 38. Perspectives on motivation (pp. 237-288). Lincoln, NE: University of Nebraska Press.

Deci, E. L., \& Ryan, R. M. (1995). Human autonomy: The basis for true self-esteem. In M. Kernis (Ed.), Efficacy, agency, and self-esteem (pp. 31-49). New York: Plenum.

Ekkekakis, P., Hall, E. E., \& Petruzzello, S. J. (2005). Variation and homogeneity in affective responses to physical activity of varying intensities: An alternative perspective on dose-response based on evolutionary considerations. Journal of Sports Sciences, 23, 477-500.

Fields, J., Murphey, M., Horodyski, M., \& Stopka, C. (1995). Factors 
associated with adherence to sport injury rehabilitation in college-age athletes. Journal of Sport Rehabilitation, 4, 172-180.

Fisher, A. C., Domm, M. A., \& Wuest, D. A. (1988). Adherence to sport injury rehabilitation programs. Physician and Sports Medicine, 16, 4750.

Folkman, S. (1984). Personal control and stress and coping processes: A theoretical analysis. Journal of Personality and Social Psychology, 46, $839-852$

Frankel, R. M. (1995). Emotion and the physician-patient relationship. Motivation and Emotion, 19, 163-173.

Garrity, T. F. (1981). Medical compliance and the clinician-patient relationship: A review. Social Science and Medicine, 15, 215-222.

Haynes, R. B. (1976). A critical review of the determinants of patient compliance with therapeutic regimens. In D. L. Sackett \& R. B. Haynes (Eds.), Compliance with therapeutic regimens (pp. 127-145). Baltimore: Johns Hopkins University Press.

Haynes, R. B., McKibbon, K. A., \& Kanani, R. (1996). Systematic review of randomized trials of interventions to assist patients to follow prescriptions for medications. Lancet, 348, 383-386.

Kim, S. S., Kaplowitz, S., \& Johnston, M. V. (2004). The effects of physician empathy on patient satisfaction and compliance. Evaluation \& the Health Professions, 27, 237-251.

Levy, A. R., Polman, R. C. J., Clough, P., \& McNaughton, L. R. (2006). Adherence to sport injury rehabilitation programmes: A conceptual review. Research in Sports Medicine: An International Journal, 14, $149-162$.

McAuley, E., Shaffer, S. M., \& Rudolph, D. (1995). Affective responses to acute exercise in elderly impaired males: The moderating effects of self-efficacy and age. International Journal of Aging and Human Development, 41, 13-27.

Meichenbaum, D., \& Turk, D. C. (1987). Facilitating treatment adherence: A practitioner's guidebook. New York: Plenum.

Newton, B. W., Savidge, M. A., Barber, L., Cleveland, E., Clardy, J., Beeman, G., et al. (2000). Differences in medical students' empathy. Academic Medicine, 75, 1215.

Niven, A. (2007). Rehabilitation adherence in sport injury: Sport physiotherapists' perceptions. Journal of Sport Rehabilitation, 16, 93-110.

Reis, H. T., \& Franks, P. (1994). The role of intimacy and social support in health outcomes: Two processes or one? Personal Relationships, 1, $185-197$.

Shaw, T., Williams, M. T., \& Chipchase, L. S. (2005). A review and user's guide to measurement of rehabilitation adherence following anterior cruciate ligament reconstruction. Physical Therapy in Sport, 6, 45-51.

Sheldon, K. M., Williams, G. C., \& Joiner, T. (2003). Self-determination theory in the clinic: Motivating physical and mental health. New Haven, CT: Yale University Press.

Silvester, J., Patterson, F., Koczwara, A., \& Ferguson, E. (2007). "Trust me": Psychological and behavioral predictors of perceived physician empathy. Journal of Applied Psychology, 92, 519-527.

Skinner, E., \& Edge, K. (2002). Self-determination, coping, and development. In E. L. Deci \& R. M. Ryan (Eds.), Handbook of self-determination research (pp. 297-337). Rochester, NY: The University of Rochester Press.

Taylor, A. H., \& Marlow, C. (2001). Creating an environment for recovery. In J. Crossman (Ed.), Coping with injuries: Psychological strategies for rehabilitation (pp. 103-124). Oxford, UK: Oxford University Press.

Taylor, A. H., \& May, S. (1996). Threat and coping appraisal as determinants of compliance with sports injury rehabilitation: An application of protection motivation theory. Journal of Sport Sciences, 14, 471-482.

Weiss, M. R. (2003). Psychological aspects of sport-injury rehabilitation: A developmental perspective. Journal of Athletic Training, 38, 172-175.

Williams, G. C., \& Deci, E. L. (1996). Internalization of biopsychological values by medical students: A test of self-determination theory. Journal of Personality and Social Psychology, 70, 767-779.

Williams, G. C., Gagné, M., Ryan, R. M., \& Deci, E. L. (2002). Supporting autonomy to motivate smoking cessation: A test of self-determination theory. Health Psychology, 21, 40-50.

Williams, G. C., Grow, V. M., Freedman, Z. R., Ryan, R. M., \& Deci, E. L. (1996). Motivational predictors of weight loss and weight-loss maintenance. Journal of Personality and Social Psychology, 70, 115-126.

Williams, G. C., McGregor, H. A., Levesque, C., Kouides, R. W., Ryan, R. M., \& Deci, E. L. (2006). Testing the self-determination theory intervention for motivating tobacco cessation: Supporting autonomy and competence in a clinical trial. Health Psychology, 25, 91-101.

Williams, G. C., Rodin, G. C., Ryan, R. M., Grolnick, W. S., \& Deci, E. L. (1998). Autonomous regulation and adherence to long-term medical regimens in adult outpatients. Health Psychology, 17, 269-276.

Woodrow, K. M., Friedman, G. D., Siegelaub, M. S., \& Collen, M. F. (1972). Pain tolerance: Differences according to age, sex and race. Psychosomatic Medicine, 34, 548-556.

Zachariae, R., Pederson, C. G., Jensen, A. B., Ehrnrooth, E., Rossen, P. B., $\&$ von de Maase, H. (2003). Association of perceived physician communication style with patient satisfaction, distress, cancer-related selfefficacy, and perceived control over the disease. British Journal of Cancer, 88, 658-665.

Received June 29, 2007

Revision received October 30, 2007 Accepted November 1, 2007 